\title{
Self-Reporting PNA/DNA Primers for PCR Analysis
}

\author{
Mark J. Fiandaca, Jens J. Hyldig-Nielsen, Brian D. Gildea, and James M. Coull ${ }^{1}$ \\ Boston Probes, Bedford, Massachusetts 01730, USA
}

\begin{abstract}
We report a new fluorogenic method for sealed-tube PCR analysis using a quencher-labeled peptide nucleic acid (Q-PNA) probe. The Q-PNA hybridizes to a complementary tag sequence located at the $5^{\prime}$ end of a $5^{\prime}$ fluorophore-labeled oligonucleotide primer, quenching the primer's fluorescence. Incorporation of the primer into a doublestranded amplicon causes displacement of the Q-PNA such that the fluorescence of the sample is a direct indication of the amplicon concentration. The Q-PNA is able to quench multiple primers bearing distinct $5^{\prime}$ fluorophores in a single reaction. We show realtime quantitative detection of a single-copy gene, $K$-ras, from human genomic DNA, as well as an endpoint multiplex assay for Chlamydia trachomatis and Neisseria gonorrhoeae targets. Because the Q-PNA may be used to quench any primer that contains the $5^{\prime}$ tag sequence, it is possible to inexpensively adapt an existing primer set for use in a self-reporting fluorescent assay by including the tag sequence in one of the primers.
\end{abstract}

Recent advances in the development of self-reporting (homogeneous) amplification assays have led to the commercial availability of fluorometer-equipped thermocyclers that monitor PCR reactions in realtime, thus obviating the need for postreaction analysis of amplicons by gel electrophoresis or other techniques. In addition to labor and time savings, appropriate standards allow quantitation of starting copy number for the purposes of viral-load measurement and mRNA expression analysis. An additional benefit is the prevention laboratory contamination by amplification products, because reaction tubes are never opened.

Molecular beacons (Tyagi and Kramer 1995), Scorpions (Whitcombe et al. 1999), and Amplifluor primers (Nazarenko et al. 1997) are examples of oligonucleotide probe/primer constructs that have been used for realtime PCR analysis. These molecules have a fluorophore and quencher placed at their termini and/or located within their nucleotide sequence. The quencher is typically a nonfluorescent aromatic moiety such as 4-([4-(dimethylamino)-phenyl)-azo)-benzoic acid (DABCYL) or a heterocycle that is capable of masking the fluorescent properties of the fluorophore due to resonance energy transfer or "contact quenching" (Yaron et al. 1979). The probes are designed to adopt a hairpin structure that places the donor and acceptor moieties in close proximity to maximize the quenching effect. Separation of the dye pair due to the hybridization of the probe to its complementary amplicon (molecular beacon, Scorpion) or copyback of the extended quenched hairpin primer (Amplifluor), results in a fluorescent signal that is directly proportional to

\section{TCorresponding author.}

E-MAIL jcoull@bostonprobes.com; FAX 781-276-4931.

Article and publication are at www.genome.org/cgi/doi/10.1101/ gr.170401. the quantity of amplicon produced. Other methods such as TaqMan (Holland et al. 1991) and DzyNA-PCR (Todd et al. 2000) utilize fluorescent resonance energy transfer (FRET) oligonucleotides as substrates that are cleaved by the exonuclease activity of the Taq polymerase or a PCR-generated DNAzyme, respectively. Cleavage results in separation of the FRET pair and an increase in fluorescent signal that is indicative of the quantity of amplicon generated during amplification.

The synthesis and purification of high-quality multilabeled FRET oligonucleotides are often neither trivial nor inexpensive relative to the ease and cost of production of 5'-labeled fluorescent probes and primers. This prompted us to consider whether an oligonucleotide bearing a single 5' fluorescent label could be hybridized with a generic quencher probe and used as a primer for realtime PCR according to Figure 1. The approach would obviate the need to design, order, store, and pipette any locus-specific reagents other than primers for each target of interest. For the quencher probe, several attributes of PNA were attractive, including the high stability of short PNA/DNA hybrids (Egholm et al. 1993), the excellent thermal and enzymatic robustness of the PNA molecule (Demidov et al. 1994), and the inability of PNA to be extended or copied by polymerases (Hyrup and Nielsen 1996).

A 13-residue quencher-labeled peptide nucleic acid (Q-PNA) bearing a C-terminal DABCYL group was synthesized and hybridized to various 5' fluorophorelabeled oligonucleotides that contained a common $5^{\prime}$ Q-PNA complementary region and $3^{\prime}$ target-specific primer sequences. We confirmed the ability of the DABCYL residue to quench a number of different $5^{\prime}$ fluorescent labels in the Q-PNA/primer duplex, including 6-carboxyfluorescein, 6-carboxy-X-rhodamine, and Cy3. The length of the Q-PNA was chosen so that 

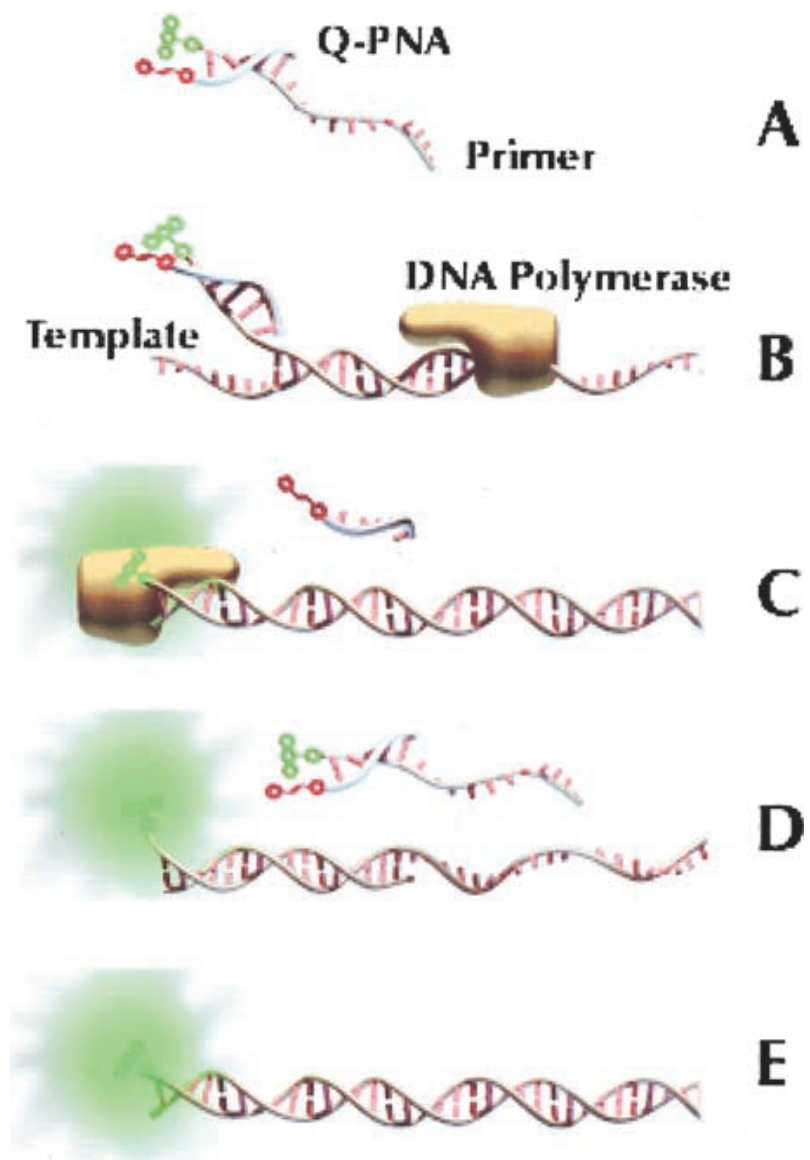

Figure 1 Schematic of the Q-PNA PCR principle. (A) Quencherlabeled Q-PNA hybridized to the 5' tag sequence of a fluorlabeled forward primer to quench the fluorescence of the primer. The $T_{m}$ of the Q-PNA/primer duplex is between the temperatures of the PCR annealing and extension steps. $(B)$ In the early rounds of thermocycling, the $3^{\prime}$ end of the forward primer hybridizes to denatured target and DNA polymerase extends the primer. (C) The reverse primer (not shown) initiates transcription of the reverse strand, resulting in displacement of the Q-PNA and incorporation of the 13-base Q-PNA-binding region into the amplicon. $(D)$ In subsequent annealing steps, the forward primer anneals fully to the reverse strand, reporting its presence for realtime analysis because excess primer is quenched by the Q-PNA. $(E)$ The end product of the reaction is a doublestranded amplicon, of which one strand is fluorescently labeled.

the Q-PNA/primer duplex had a melting temperature $\left(T_{m}\right)$ higher than the primer annealing temperature and lower than the $\mathrm{T}_{m}$ of the primer/amplicon duplex. As a result, excess primer was quenched at the annealing temperature and the fluorescence measured during the annealing step indicated the amount of primer hybridized to amplicon, plus any full-length doublestranded amplicon. Published algorithms were used to estimate DNA (SantaLucia et al. 1996) and PNA (Giesen et al. 1998) melting temperatures for the purpose of primer and Q-PNA design, and the calculated $T_{m} \mathrm{~S}$ were generally in agreement with measured values.

\section{RESULTS}

\section{Realtime Detection}

The results of triplicate realtime analysis of the singlecopy human $\mathrm{K}$-ras gene as a function of template copy number using a Q-PNA/DNA primer are shown in Figure 2. The annealing and extension temperatures of the PCR cycle were $56^{\circ} \mathrm{C}$ and $74^{\circ} \mathrm{C}$, respectively. The calculated $T_{m}$ values of the components were Q-PNA/ primer duplex, $69^{\circ} \mathrm{C}$; forward primer/target (priming region only), $61^{\circ} \mathrm{C}$; forward primer/amplicon (full length), $77^{\circ} \mathrm{C}$; and reverse primer/target, $62^{\circ} \mathrm{C}$. The cutoff threshold (Ct, the $\Delta \mathrm{Rn}$ value that establishes a positive response) was automatically set by the instrument software. The plot of Ct versus input genomes showed a sensitivity of $\sim 10$ genomes and regression analysis of the best-fit line yielded a correlation coefficient $\left(r^{2}\right)$ of 0.989. At one genome, one of three samples did not achieve the threshold value and when less than one genome was amplified, only one of six samples gave a positive response. None of the three no-target samples reached the threshold value. Analysis of reaction products by electrophoresis and Southern blotting with a $K$-ras-specific PNA probe revealed that reactions with Ct values of $\leq 56$ (i.e., $>10$ genomes) produced amplicons of the correct size and sequence, whereas reactions with Ct values of $>56$ yielded mainly primer dimers (data not shown).

\section{Multiplex Endpoint Detection}

Use of the Q-PNA for multicolor multiplex endpoint PCR analysis was then explored. We reasoned that endpoint analysis would be possible because the ultimate product of the reaction would be a fluorescent fulllength doublestranded amplicon, whereas excess labeled primers would be quenched. To this end, two sets of bacterial specific primers were designed for detection of the genitourinary pathogens Chlamydia trachomatis and Neisseria gonorrhoeae. Each primer set contained one primer that had both the Q-PNA tag sequence and a $5^{\prime}$ fluorescent label (rhodamine for $C$. trachomatis, fluorescein for $\mathrm{N}$. gonorrhoeae). The calculated $T_{m}$ values of the components were as follows: Q-PNA/primer duplex, $67^{\circ} \mathrm{C}$; forward primer/target (priming region only), $75^{\circ} \mathrm{C}$; forward primer/amplicon (full length), $85^{\circ} \mathrm{C}$; and reverse primer/target, $72^{\circ} \mathrm{C}$.

The primer sets were added to PCR reactions with various genomic DNA targets. As shown in Figure 3, fluorescent color following PCR was dependent on the addition of specific bacterial templates to the reaction tubes. Gel analysis of products from positive reactions confirmed that amplicons generated were the correct length, whereas negative controls produced nearbackground levels of fluorescence and no bands. Samples that contained both N. gonorrhoeae and C. trachomatis DNA, resulted in reactions having an orange- 

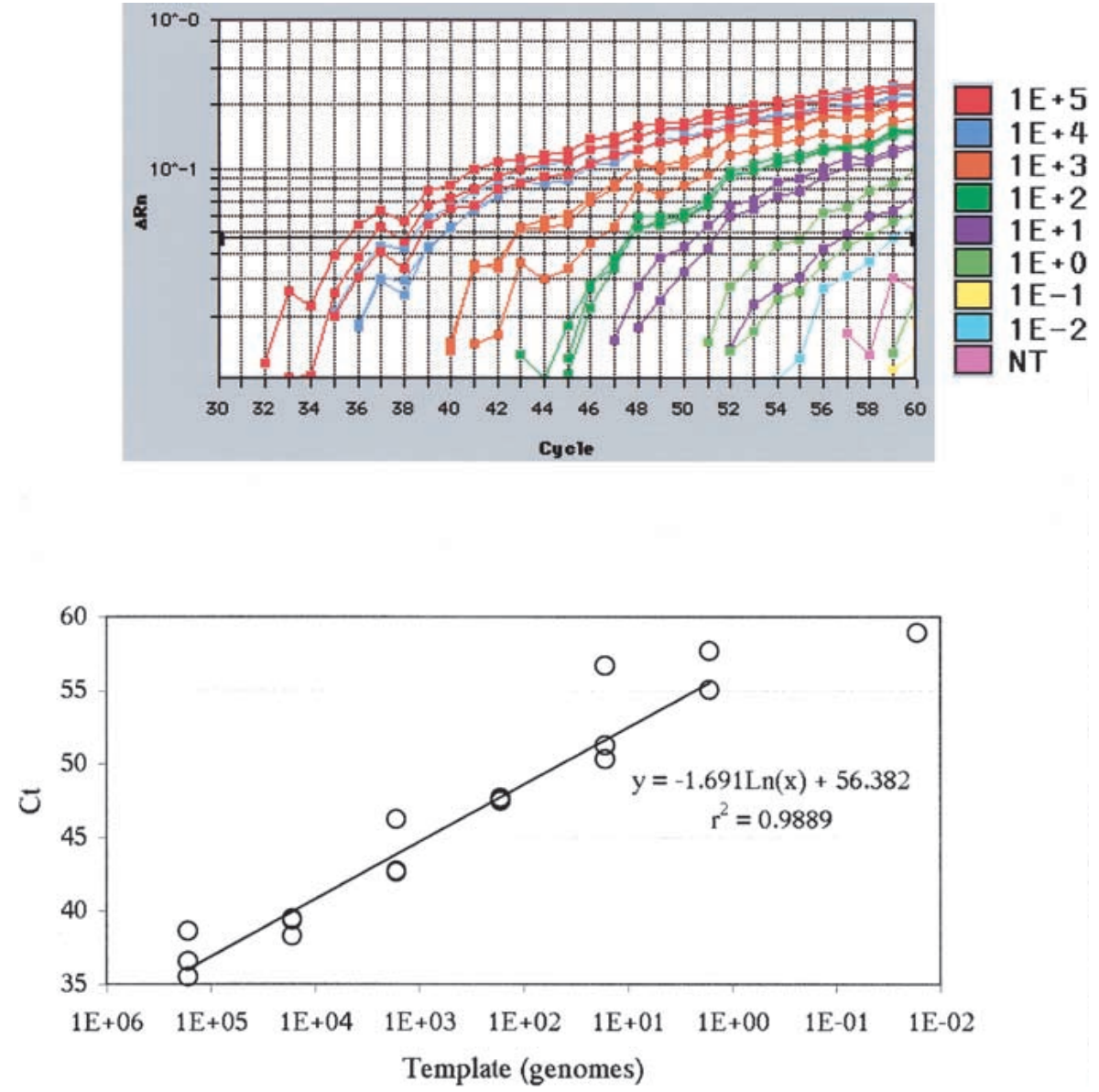

Figure 2 (A) Results from amplifications of the human $K$-ras gene. Tenfold serial dilutions of human DNA comprising $10^{5}-10^{-2}$ genomes per reaction, as well as no template controls (NT) were thermocycled and fluorescence was recorded during the annealing step of each cycle. The $\Delta \mathrm{Rn}$ values represent fluorescence at each cycle, minus the average baseline signal calculated between cycles 3-15, as generated by the default settings on the $A B I 7700$ instrument. (B) A plot of cutoff threshold (Ct) value vs. input template concentration.

yellow color, consistent with the presence of both targets. For each tube, the fluorescent signals were measured and the values obtained were normalized to the fluorescence of the same quantity of labeled primer in the absence of the Q-PNA. This normalization allowed us to represent PCR-generated fluorescence as a percent of the maximum potential signal from each primer. As shown in Figure 3C, $>20 \%$ of the primers were incorporated into amplicons in the positive reactions.

\section{DISCUSSION}

This simple, easy-to-implement, robust, inexpensive method that we have developed for homogeneous detection of PCR products is suitable for both realtime and endpoint analysis. It utilizes a generic Q-PNA reagent to quench fluorescent signal from nonincorporated PCR primers. In cases where endpoint PCR is sufficient to analyze samples of interest, reaction analysis using the Q-PNA requires nothing more than a UV light source and the naked eye. With this simple setup, we have shown it is possible to analyze samples for at least two different targets at the same time using a single Q-PNA and multiple primers bearing distinct labels. The use of a fluorescent plate reader allows the technique to be further multiplexed to include as many primer pairs as there are independently detectable fluorescent labels.

For realtime quantitative PCR applications such as expression analysis and gene dosage determination, we have shown that our system monitors generation of amplicons through an increase in fluorescence that correlates directly with input template concentration. The sensitivity and linearity of realtime detection using the Q-PNA is comparable to results obtained by others using TaqMan probes to detect amplification of single-copy sequences from human genomic DNA (Heid et al. 1996).

The length of the Q-PNA was chosen to be suitable for both realtime and endpoint PCR assays. Thus, the 13-mer PNA described here hybridized with sufficient strength to provide rapid and efficient quenching of excess primer at the temperature used for primer an- 

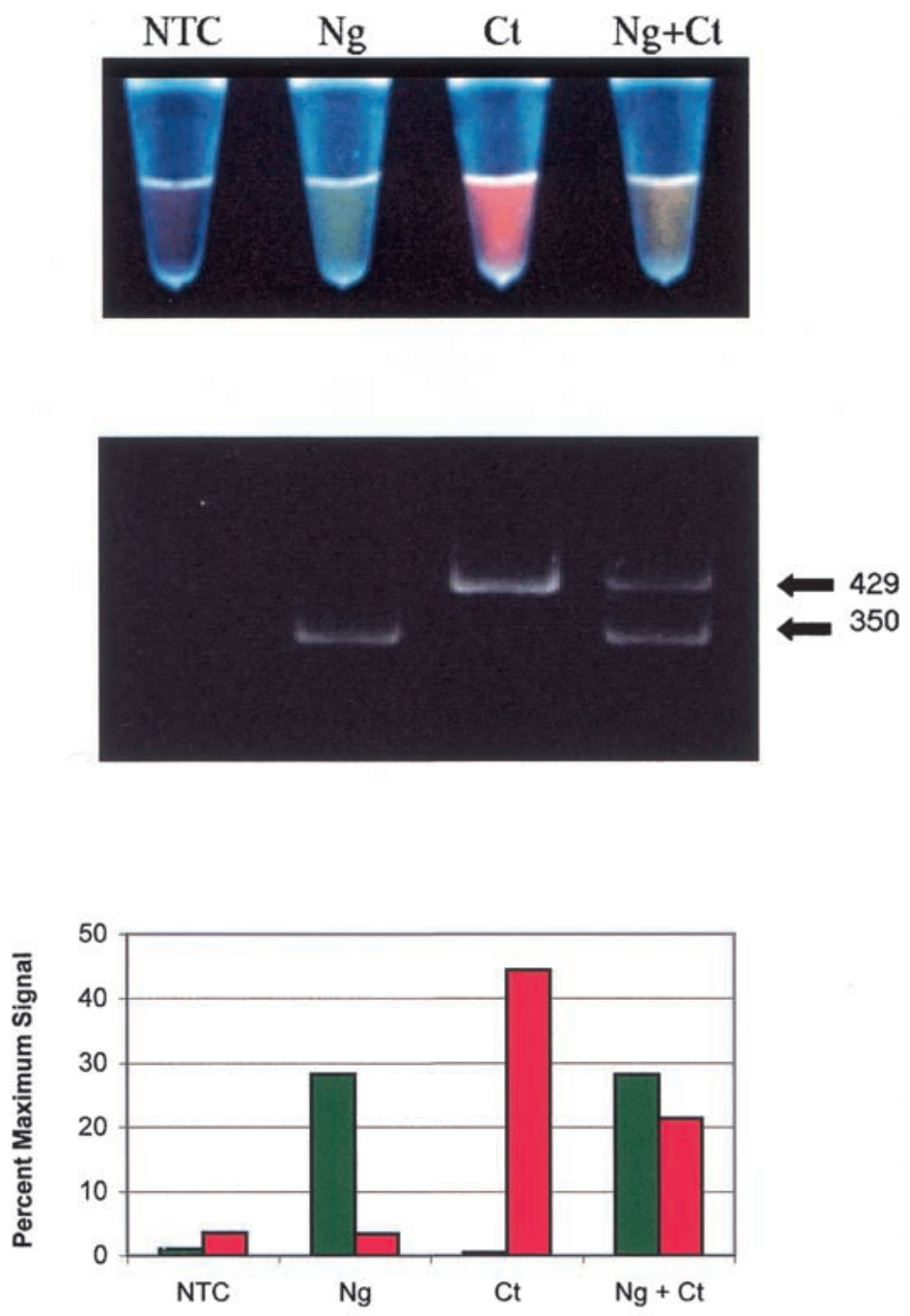

Fluorescein

\section{Rhodamine}

Figure 3 (A) Post-PCR photograph of multiplexed bacterial samples. From left to right, the tubes contained no template control (NTC), $10^{4}$ copies Neisseria gonorrhoeae DNA (Ng), $10^{4}$ copies Chlamydia trachomatis DNA $(\mathrm{Ct})$, and $10^{4}$ copies of both N. gonorrhoeae DNA and C. trachomatis DNA $(\mathrm{Ng}+\mathrm{Ct})$. The tubes were illuminated from below with a 302-nm UV light, a wavelength that stimulated visible fluorescence from both dye labels. ( $B$ ) The reaction products were analyzed by polyacrylamide gel electrophoresis. Two sizes of bands were visible in the UV-irradiated gel, corresponding to $429 \mathrm{bp}$ and $350 \mathrm{bp}$. Although not visible in this black-and-white photograph, the 429-bp band fluoresced a bright red color and the 350-bp band was bright green. (C) The fluorescent signal of each reaction following PCR. Reaction fluorescence was normalized against the starting fluorescence of the primer in the absence of the Q-PNA.

nealing $\left(56^{\circ} \mathrm{C}-60^{\circ} \mathrm{C}\right)$ during realtime PCR. However, for endpoint measurements that are made at room temperature post-PCR, we have successfully utilized QPNAs as short as nine bases $\left(T_{m}=\sim 40^{\circ} \mathrm{C}\right)$. It is also possible to construct the system with the labels reversed (i.e., a fluorescein-labeled PNA and a DABCYL-labeled primer). This change in label polarity monitors the generation of free (fluorescent) PNA and allows for use of significantly less PNA probe; however, the sensitivity of the system is not as great.

We have constructed more than 10 primer sets, all of which performed in a similar manner to those presented above. DABCYL was an effective quencher for a wide array of fluorescent labels, which further simplified the Q-PNA design. During the initial development of the method, we occasionally observed the formation of nontemplated products (i.e., primer dimers), which led to fluorescence in the absence of amplicon generation, we were able to eliminate this by utilizing hot-start techniques to prevent side reactions during PCR setup. Thus, we recommend use of one of the many commercially available thermally activated polymerases to prevent primer dimer formation, especially when performing multiplexed reactions.

Q-PNA/primers may be combined with other PCR-monitoring and specificity-enhancing techniques. For example, the use of PNA-based PCR clamping (Ørum et al. 1993) in combination with the method described here may provide a closed-tube method for performing highly sensitive point mutation and SNP analysis. The QPNA might also be employed in the ARMS system (Newton et al. 1989), which relies on singly and doubly 3' mismatched primers for selective amplification of point mutations. Additionally, combining the Q-PNA approach with other technologies such as TaqMan or molecular beacons may increase the amount of information that can be garnered from a single PCR reaction. Finally, although not yet explored, we believe the Q-PNA tag sequence within the primer can be designed to incorporate restriction sites or affinity handles resulting in amplicons that can be easily isolated, detected, or cloned using generic reagents.

\section{METHODS}

\section{Realtime PCR}

Thermocycling reactions were performed using the ABI PRISM 7700 (PE Applied Biosystems) and analyzed with the accompanying software. TaqMan Universal PCR Master Mix, supplied as a $2 \times$ solution (PE Biosystems) containing all components needed for PCR, except primers and template, was used to formulate reactions having total volumes of $50 \mu \mathrm{L}$. The primers $(0.9 \mu \mathrm{M})$ were 5'-6-carbxyfluorescein-TCA TTC GCA ATC ACT CTA TTG TTG GAT CAT ATT-3' (forward) and 5'-ATG ACT GAA TAT AAA CTT GT-3' (reverse). The Q-PNA $(1.35 \mu \mathrm{M})$ was $\mathrm{N}^{\prime}$ TGA TTG CGA ATG A-(DABCYL)-C' (Boston Probes) and was stored in $\mathrm{N}, \mathrm{N}^{\prime}$-dimethylformamide (DMF)/water $(1 / 1, \mathrm{v} / \mathrm{v})$, resulting in a final DMF concentration of $0.2 \% \mathrm{v} / \mathrm{v}$ in the PCR reactions. Human genomic DNA stock solution (Sigma) was 
diluted in water and added to the PCR tube immediately prior to thermocycling. The PCR cycle was as follows: $50^{\circ} \mathrm{C}\left(120^{\prime \prime}\right)$, $95^{\circ} \mathrm{C}\left(600^{\prime \prime}\right)$, followed by 60 rounds of $95^{\circ} \mathrm{C}\left(30^{\prime \prime}\right), 56^{\circ} \mathrm{C}\left(30^{\prime \prime}\right)$, $74^{\circ} \mathrm{C}\left(30^{\prime \prime}\right)$.

\section{Multiplex PCR}

Reactions were performed in a GeneAmp 2400 (PE Applied Biosystems). PCR reactions contained $2.5 \mathrm{mM} \mathrm{MgCl}_{2}, 250 \mu \mathrm{M}$ NTPs, 1.5 units AmpliTaq DNA polymerase, $50 \mathrm{mM} \mathrm{KCl}$, and $10 \mathrm{mM}$ TRIS at pH 8.3 (PE Applied Biosystems). Each reaction also contained two fluor-labeled DNA primers $(0.2 \mu \mathrm{M})$ and two nonlabeled primers $(0.25 \mu \mathrm{M})$. The primers were as follows:N. gonorrhoeae: 5'-6-carboxyfluorescein-TCA TTC GCA ATC AAC CCA CGC TTT CGG ACA T-3' (forward), 5'-GTC AGG GAA GAA AAG GCT GTT-3' (reverse); C. trachomatis: 5'-6-carboxy-X-rhodamine-TCA TTC GCA ATC AAC GGA TGG GGT TGA GAC C-3' (forward), 5'-CGC TTG GGA ATA AGA GAA GAC-3' (reverse). Each reaction contained the 13mer Q-PNA as described above $(0.6 \mu \mathrm{M})$, resulting in a final DMF concentration of $0.6 \%, \mathrm{v} / \mathrm{v}$. At the beginning of thermocycling, tubes were heated for $30 \mathrm{sec}$ at $95^{\circ} \mathrm{C}$, at which point $1 \mu \mathrm{L}$ of DNA polymerase solution was added to each hot reaction, for a total volume of $30 \mu \mathrm{L}$. Thermocycling proceeded for 35 rounds: $95^{\circ} \mathrm{C}\left(10^{\prime \prime}\right), 60^{\circ} \mathrm{C}\left(20^{\prime \prime}\right), 75^{\circ} \mathrm{C}\left(20^{\prime \prime}\right.$, with a $75^{\circ} \mathrm{C}$ $\left(300^{\prime \prime}\right)$ extension step at the end of the final round. The primer sets were designed to amplify similar yet species-specific regions of the rRNA genes of the bacterial genomes. Amplicons generated by the $N$. gonorrhoeae and $C$. trachomatis primer sets were $350 \mathrm{bp}$ and $429 \mathrm{bp}$, respectively. Positive samples were spiked with $1 \mu \mathrm{L}$ total genomic DNA from $N$. gonorrhoeae and/ or $C$. trachomatis, both at a concentration $1 \times 10^{4}$ copies $/ \mu \mathrm{L}$ (Advanced Biotechnologies). The control sample was spiked with $1 \mu \mathrm{L}$ water. Post-PCR, the tubes were photographed over a UV transilluminator using Kodak Max 800 film $\left(0.25^{\prime \prime}\right.$, f1.8). The photograph was scanned and adjusted to enhance brightness and contrast. A $15-\mu \mathrm{L}$ portion of each reaction was added to $35 \mu \mathrm{L} 0.1 \mathrm{M}$ TRIS-Cl at $\mathrm{pH} 9.0$ in an ELISA plate and read in a Victor Multilabel counter (Wallac). Each well was examined for the characteristic fluorescent emissions of both fluorescein and rhodamine. An additional $12 \mu \mathrm{L}$ of each reaction was combined with $3 \mu \mathrm{L} 5 \times$ loading dye and analyzed on a $4 \%-$ $20 \%$ gradient polyacrylamide gel. The unstained gel was photographed with UV transillumination using Polaroid film $\left(1.0^{\prime \prime}, \mathrm{f} 4.5\right)$. The gel was subsequently stained with a fluorescent intercalating dye to visualize a DNA size marker run in an adjacent lane (not shown).

The publication costs of this article were defrayed in part by payment of page charges. This article must therefore be hereby marked "advertisement" in accordance with 18 USC section 1734 solely to indicate this fact.

\section{REFERENCES}

Demidov, V.V., Potaman, V.N., Frank-Kamenetskii, M.D., Egholm, M., Buchardt, O., Sönnichsen, S.O., and Nielsen, P.E. 1994. Stability of peptide nucleic acids in human serum and cellular extracts. Biochem. Pharma. 48: 1310-1313.

Egholm, M., Buchardt, O., Christensen, L., Behrens, C., Freier, S.M., Driver, D.A., Berg, R.H., Kim, S.K., Norden, B., and Nielsen, P.E. 1993. PNA hybridizes to oligonucleotides obeying Watson-Crick hydrogen bonding rules. Nature 365: 566-568.

Giesen, U., Kleider, W., Berding, C., Geiger, A., Ørum, H., and Nielsen, P.E. 1998. A formula for thermal stability $\left(T_{m}\right)$ prediction of PNA/DNA duplexes. Nucleic Acids Res. 26: 5004-5006.

Heid, C.A., Stevens, J., Livak, K.J., and Williams, P.M. 1996. Real Time Quantitative PCR. Genome Res. 6: 986-994.

Holland, P.M., Abramson, R.D., Watson, R., and Gelfand, D.H. 1991. Detection of specific polymerase chain reaction product by utilizing the 5'- 3' exonuclease activity of Thermus aquaticus DNA polymerase. Proc. Natl. Acad. Sci. 88: 7276-7280.

Hyrup, B. and Nielsen, P.E. 1996. Peptide nucleic acids (PNA): Synthesis, properties and potential applications. Bioorg. Med. Chem. 4: 5-23.

Nazarenko, I.A., Bhatnagar, S.K., and Hohman, R.J. 1997. A closed tube assay format for amplification and detection of DNA based on energy transfer. Nucleic Acids Res. 25: 2516-2521.

Newton, C.R., Graham, A., Heptinstall, L.E., Powell, S.J., Summers, C., Kalsheker, N., Smith, J.C., and Markham, A.F. 1989. Analysis of any point mutation in DNA. The amplification refractory mutation system (ARMS). Nucleic Acids Res. 17: 2503-2516.

Ørum, H., Nielsen, P.E., Egholm, M., Berg, R.H., Buchardt, O., and Stanley, C. 1993. Single base pair mutation analysis by PNA directed PCR clamping. Nucleic Acids Res. 21: 5332-5336.

SantaLucia Jr., J., Allawi, H.T., and Seneviratne, P.A. 1996. Improved nearest-neighbor parameters for predicting DNA duplex stability. Biochemistry 35: 3555-3562.

Todd, A.V., Fuery, C.J., Impey, H.L., Applegate, T.L., and Haughton, M.A. 2000. DzyNA-PCR: Use of DNAzymes to detect and quantify nucleic acid sequences in a real-time fluorescent format. Clin. Chem. 46: 625-630.

Tyagi, S. and Kramer, F.R. 1995. Molecular beacons: Probes that fluoresce upon hybridization. Nat. Biotechnol. 14: 303-308.

Whitcombe, D., Theaker, J., Guy, S.P., Brown, T., and Little, S. 1999. Detection of PCR products using self-probing amplicons and fluorescence. Nat. Biotechnol. 17: 804-807.

Yaron, A., Carmel, A., and Katchalski-Katzir, E. 1979. Intramolecularly quenched fluorogenic substrates for hydrolytic enzymes. Anal. Biochem. 95: 228-235.

Received November 11, 2000; accepted in revised form February 8, 2001.
Genome Research www.genome.org 


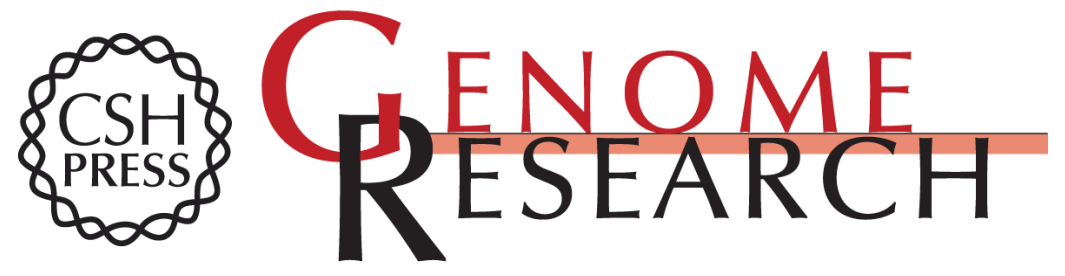

\section{Self-Reporting PNA/DNA Primers for PCR Analysis}

Mark J. Fiandaca, Jens J. Hyldig-Nielsen, Brian D. Gildea, et al.

Genome Res. 2001 11: 609-613

Access the most recent version at doi:10.1101/gr.170401

References This article cites 13 articles, 3 of which can be accessed free at:

http://genome.cshlp.org/content/11/4/609.full.html\#ref-list-1

\section{License}

Email Alerting Receive free email alerts when new articles cite this article - sign up in the box at the Service top right corner of the article or click here.

\section{Affordable, Accurate Sequencing.}

To subscribe to Genome Research go to: https://genome.cshlp.org/subscriptions 